\title{
Habilidades cognitivas promovidas en los textos escolares de Historia y Geografía en estudiantes de Educación Básica chilena
}

\section{Cognitive skills promoted in History and Geography textbooks in Chilean elementary school students}

\section{Competências cognitivas promovidas em livros de História e Geografia em estudantes chilenos do ensino básico}

\author{
Alejandro Sepúlveda Obreque \\ Universidad de Los Lagos \\ Osorno, Chile \\ asepulve@ulagos.cl \\ (D) ORCID: https://orcid.org/0000-0002-5033-8400 \\ Hernán Delgado Delgado \\ Universidad de Los Lagos \\ Osorno, Chile \\ hernan.delgado@ulagos.cl \\ (D) ORCID: https://orcid.org/0000-0002-9685-3459 \\ Alejandro Villalobos Clavería \\ Universidad de Concepción \\ Concepción, Chile \\ avillalo@udec.cl \\ (10) ORCID: https://orcid.org/0000-0003-1440-9942 \\ Sebastián Peña Troncoso \\ Universidad Austral de Chile \\ Valdivia, Chile \\ sebap988@hotmail.com \\ (D) ORCID: https://orcid.org/0000-0002-5438-0868
}

Recibido - Received - Recebido: 15 / 07 / 2021 Corregido - Revised - Revisado: 12 / 11 / 2021 Aceptado - Accepted - Aprovado: 25 / 11 / 2021

DOl: https://doi.org/10.22458/ie.v24i36.3907

URL: https://revistas.uned.ac.cr/index.php/innovaciones/article/view/3907

\begin{abstract}
Resumen: El propósito del estudio fue analizar las habilidades cognitivas que se promueve en los textos escolares de Historia y Geografía de sétimo y octavo año, así como su correspondencia con las habilidades declaradas en las Bases Curriculares. La investigación se planteó bajo los parámetros del método integrativo, el cual usa elementos del diseño hermenéutico y descriptivo, en este se recurre a la técnica de análisis documental, aplicación de estadística descriptiva, citas o fragmentos que permitieron pesquisar y procesar los datos. La media más alta, presente en los textos escolares, corresponde al tipo de habilidades creativas (57,5\%), seguida de las analíticas $(47,0 \%)$, y, finalmente, las habilidades descriptivas $(42,0 \%)$ y críticas $(25,0 \%)$. No fue posible observar diferencias importantes entre la promoción de habilidades cognitivas según nivel educativo. En octavo año las habilidades de tipo creativa y analítica suman más que en sétimo, como también se constató la presencia de mayor frecuencia de habilidades cognitivas de tipo críticas. Se puede concluir la total correspondencia entre las habilidades cognitivas declaradas en las Bases Curriculares y las actividades educativas propuestas en los textos escolares. De igual modo, queda de manifiesto que las actividades presentes en estos textos, promueven habilidades que potencian operaciones y procedimientos mentales, en los cuales se involucran procesos de creación, análisis, descripción y críticos. Las habilidades más presentes en los textos son: observar, explicar y aplicar. Las habilidades más ausentes: definir conceptos, discutir datos, promover la imaginación, formular hipótesis, representar secuencias cronológicas y proponer soluciones a problemas.
\end{abstract}

Palabras clave: habilidades cognitivas, textos escolares, ciencias sociales, enseñanza de la geografía, educación. 


\begin{abstract}
The purpose of the study was to analyze the cognitive skills promoted in History and Geography textbooks for seventh and eighth grade and their correspondence with the skills in the Curricular substructure. The research was conducted under the parameters of the integrative method, which uses elements of hermeneutics and descriptive design, using the documentary analysis technique, application of descriptive statistics, quotations, or fragments that allowed data researching and processing. The highest average, present in the school texts, corresponds to the type of creative skills (57.5\%), followed by analytical skills (47.0\%), and finally, descriptive skills (42.0\%) and critical skills (25.0\%). It was not possible to observe significant differences between the promotion of cognitive skills according to educational level. In the eighth grade, creative and analytical skills were more frequent than in seventh grade, as was the presence of a greater frequency of critical cognitive skills. It can be concluded that there is a complete correspondence between the cognitive skills of the Curricular substructure and the educational activities proposed in the school textbooks. Likewise, the activities in these texts promote mental operations and procedures, which involve creative, analytical, descriptive, and critical processes. The skills most present in the texts are: observing, explaining, and applying. The most absent skills are: defining concepts, discussing data, promoting imagination, formulating hypotheses, representing chronological sequences, and proposing solutions to problems.
\end{abstract}

Keywords: cognitive skills, school texts, social sciences, geography teaching, education.

Resumo: $O$ objetivo do estudo era analisar as competências cognitivas promovidas nos manuais de História e Geografia para estudantes da sétima e oitava série, bem como a sua correspondência com as competências declaradas nos Currículos de Base. A pesquisa foi realizada sob os parâmetros do método integrativo, que utiliza elementos de concepção hermenêutica e descritiva, utilizando a técnica de análise documental, aplicação de estatísticas descritivas, citações ou fragmentos que permitiram pesquisar e processar os dados. A média mais elevada, presente nos textos escolares, corresponde à categoria de competências criativas (57,5\%), seguida de competências analíticas (47,0\%), e por fim, competências descritivas (42,0\%) e competências críticas (25,0\%). Não foi possível observar diferenças significativas entre a promoção das capacidades cognitivas conforme o nível educacional. Na oitava série, as competências criativas e analíticas eram mais comuns do que na sétima, tal como a presença de uma maior frequência de competências cognitivas críticas. Pode-se concluir que existe uma correspondência completa entre as competências cognitivas declaradas nas bases curriculares e as atividades educativas propostas nos manuais escolares. Do mesmo modo, é evidente que as atividades presentes nestes textos promovem competências que promovem operações e procedimentos mentais, nos quais estão envolvidos processos de criação, análise, descrição e crítica. As competências mais presentes nos textos são: observar, explicar e aplicar. As competências mais ausentes: definir conceitos, discutir dados, promover a imaginação, formular hipóteses, representar sequências cronológicas e propor soluções para os problemas.

Palavras-chave: competências cognitivas, textos escolares, ciências sociais, ensino da geografia, educação.

\title{
INTRODUCCIÓN
}

Las habilidades cognitivas se avocan preferentemente a las destrezas y los procesos que permiten adquirir el conocimiento de diversas maneras y bajo múltiples perspectivas, las cuales tienen como herramientas a los sentidos, la experiencia y la vivencia en el estudiantado, y provocan el aprendizaje. Según Portillo (2017), las habilidades tienen su fundamento en lo que las personas son capaces de hacer desde sus condiciones neurofisiopsicológicas y estas pueden ser específicas, cuando ciertas tareas se realizan en situaciones complejas. No existe una única definición sobre qué es una habilidad; pues este es un constructo social. Por cierto, este término se ha utilizado en diferentes países y con diferentes enfoques conceptuales y disciplinarios, según sea el propósito investigativo donde se va a aplicar, ya sea lo psicológico, sociológico, comunicacional, entre otros.

Para lograr las habilidades descriptivas, analíticas, críticas o creativas resulta necesario potenciarlas con el insumo disciplinar que se pretenda facilitar, compartir y profundizar. Esto último va de la mano con la tarea que efectúa la persona docente, debido a que es él o ella quien asume esa responsabilidad, pero no solo desde lo teórico-disciplinar; sino también transita desde lo ético hasta lo material y lo concreto (Sáiz, 2011).

El desafío, por tanto, está puesto en el ejercicio constante y permanente que los docentes efectúan, de manera cotidiana, para tratar de encauzar, potenciar o transmitir estas habilidades cognitivas. Romero y Tapia (2014) expresan que, para adquirir una habilidad cognitiva, se deben seguir tres pasos: reconocimiento, desarrollo y capacidad para que se utilice autónomamente en un momento posterior o futuro. 
Todo esto puede ir tras habilidades más básicas o de procedimiento o investigativas o de dominio afectivo (Chona et al., 2006).

Los libros de textos escolares, que es el objeto de estudio de esta investigación, según Sáiz (2011), se constituyen en el recurso educativo fundamental de los docentes de Geografía e Historia. Es un instrumento elemental en el aprendizaje de los estudiantes.

Pagés (2008); Martínez, Valls, Pineda (2009) plantean tres grandes visiones sobre el libro de texto. Una minoría crítica de docentes considera que el manual escolar ya no es una herramienta útil, y, por tanto, recurriría a confeccionar sus propios materiales o emplear los textos opcionales, editados por grupos de innovación didáctica. Otro sector de los docentes, juzga que el libro de texto todavía puede dar buenos resultados con un uso crítico y reflexivo, siempre y cuando se seleccionen y combinen con otros materiales propios u opcionales, $y$, un tercer grupo de profesores, quienes en su mayoría, por las circunstancias que sean, efectúan un uso fiel, envolvente y casi único del libro de texto.

Según Sáez (2017) las investigaciones que abordan la enseñanza de la Historia en los libros de texto en Chile, concluyen que prima una visión episódica y memorística, la cual se podría relacionar con la escasez de preguntas de nivel cognitivo superior.

Las actividades de los textos de Historia de primero y segundo nivel de Educación Secundaria Obligatoria (ESO) guardan una difícil relación con el desarrollo de destrezas cognitivas de los estudiantes. Estos recursos, apelan en su mayoría a las actividades que demandan un nivel cognitivo poco exigente, básicamente recordar o reproducir información factual y conceptual. Las actividades giran en torno a recordar-reproducir lo enunciado y transmitido en el texto académico (Sáiz, 2011).

El caso de los textos escolares (Landa et al. 2019a y 2019b) que abordan la materia de Historia, Geografía y Ciencias Sociales de sétimo y octavo año, en comparación con los lineamientos curriculares del Ministerio de Educación de Chile (Mineduc), evidencian una concordancia clara respecto a lo que se busca lograr en el estudiantado de Educación Básica. No obstante, deben apuntarse algunos elementos, los cuales permiten un acercamiento teórico a las razones que se esgrimen en torno a las disciplinas recién mencionadas; pues sus características son particulares $y$, en este estudio, constituyen el objeto que le ofrece vida. Entonces, una de las características que se debería develar de la enseñanza tradicional, radica en la reflexión sobre el pasado, erguido en el presente dinámico y controvertido -que no da certezas inequívocas- y eso está en concordancia con una habilidad cognitiva de tipo analítica, a la vez, presente en las pautas que entregan los textos escolares (Sáez-Rosenkranz, 2017).

Por lo tanto, esta disciplina trinitaria debe ceder paso no solo a su traje típico anclado al pasado, sino que debe adentrarse en las coyunturas del tiempo reciente; pues de este modo abre paso a habilidades cognitivas que el estudiantado puede lograr, afincando su conocimiento a procesos actuales, con estos no solo puede viajar en el tiempo, sino enjuiciar, criticar, discutir o detectar problemas y ahí es cuando ya se están activando habilidades cognitivas variadas: analíticas, críticas o creativas.

De acuerdo con lo anterior, las citadas habilidades promueven la transdisciplinariedad, lo que profundiza la reflexión y la integración disciplinar, integrando y complejizando los roles docentes, pero también las relaciones que establecen los discentes al momento de enfrentarse a un tema histórico, geográfico o de las ciencias sociales, al tener más herramientas para discernir o problematizar.

En el caso de las ciencias sociales -muchas veces considerada como una disciplina en sí misma-, contenedora de un cúmulo de materias unidas por la epistemología sobre la que subyace; cabe indicar que ella no hace más que promover las habilidades cognitivas que habilitan al estudiantado en áreas prioritarias para la Educación Básica y Media en Chile, dado que, según Morales (2017), con ella se potencia la formación ciudadana al permitirle a los educandos argumentar, explicar y exponer visiones diversas sobre problemas contingentes en la construcción del conocimiento, desde y a partir de una habilidad 
cognitiva crítica, a la vez que se fortalece el pensamiento crítico-evaluador, el cual permite establecer concordancias entre la producción del conocimiento, su evolución y su transformación (Morales, 2017).

Por otra parte, la enseñanza y la práctica de la disciplina histórica, se complementa con los currículos educativos nacionales al exigir la facilitación de contenidos de primer orden o sustantivos, cuando se trata de responder al ¿qué?, ¿quién, ¿cuándo? y, ¿dónde? (Gómez et al., 2014); es decir, esbozos iniciales de lo que se desea profundizar con las personas estudiantes de Educación General Básica (EGB). No obstante, también se inscriben en esta práctica los contenidos de segundo orden o estratégicos que equivalen a la aplicación de diversas capacidades, competencias que favorecen la resolución de procesos o coyunturas históricas, las cuales permiten comprender y aprehender de manera más compleja el pasado (Gómez et al., 2014). Las habilidades que se pueden trasuntar desde la experiencia del profesor de la disciplina, aluden a la búsqueda, la selección y el tratamiento de fuentes históricas, la empatía o la perspectiva histórica (Gómez et al., 2014).

Lo anterior no solo demuestra que lo rescatado por los textos escolares distribuidos en el 2019 por el Mineduc, ha decantado un tránsito comprometido hacia contenidos de segundo orden y sus respectivas habilidades cognitivas, sino también hacia una relación inequívoca con la Ley 20.911, la cual aborda un aspecto que antes se trataba de manera disciplinar y capsular: la formación ciudadana. Gracias a dicha Ley de 2016, hoy la formación ciudadana es el denominador común transversal del currículum nacional; por lo tanto, la Historia, la Geografía y las Ciencias Sociales no están solas, ni en el aspecto teórico, ni en el práctico, siendo este último el que logra brillar en el ámbito de las habilidades cognitivas creativas. La potenciación de tales habilidades parece positivamente manifiesta, cuando se aprende a hacer, pensar y reflexionar con estas disciplinas; es decir: saber, saber ser y saber hacer.

Si bien se puede estar plenamente de acuerdo con las bondades del desarrollo de las distintas y complementarias habilidades cognitivas, resulta válido señalar que el Sistema de Medición de la Calidad de la Educación (SIMCE) abarca contenidos, habilidades y actitudes, mayormente evaluados sobre las habilidades desarrolladas en los textos del estudiante. Por consiguiente, esto significa que se visualiza un amplio espectro de aprendizaje del estudiantado al que no se le observa (Godoy, 2015), pero, por sobre todo continúa fragmentando el conocer de los estudiantes; aun sabiendo que el conocimiento, desde una perspectiva sistémica y compleja (Moreno, Toro y Gómez, 2020), no es posible fraccionarlo, pero sí profundizar o ampliar (Peña, Toro, Cárcamo, Hernández-Mosqueira y Cresp, 2021).

De acuerdo con lo anterior, debería destacarse un enfoque socio-cognitivo; pues el aula de clases es vista como una actividad eminentemente cultural, donde hay interacción y aprendizaje sobre la base de una diálogo que guía el profesor (Godoy, 2015) y se promueven contenidos de primer o segundo orden.

En particular, en el caso que se plantea, las habilidades cognitivas que se destacan en la asignatura de Historia, Geografía y Ciencias Sociales oscilan entre el pensamiento espacial y temporal; el uso, el análisis y el trabajo de fuentes; la comunicación, la interacción así como el pensamiento crítico, que involucra conocimiento, acción y valoración de lo estudiado, tal como lo enfatiza Godoy (2015).

Igualmente importante de mencionar es que en el caso de las habilidades asociadas a aquella disciplina, son dos las variables importantes a considerar; una, se refiere a una mayor flexibilidad en el razonamiento, y otra, obliga al estudiantado a comprender conceptos tanto históricos como sociales y los cuales no necesariamente se hallan presentes en otras disciplinas o materias del currículo (Sáez-Rosenkranz, 2017). Esto se debe a que el discente no puede eliminar variables históricas o sociales que son concomitantes a procesos o coyunturas de esa índole, como sí podría hacerlo si tuviera que hacer un cálculo matemático o un experimento sobre volúmenes (Carretero, 2002), lo cual a todas luces hace de la Historia, la Geografía y las Ciencias Sociales un conjunto disciplinar rico, profundo y germinal de habilidades cognitivas que actúan de forma conjunta y paralela. 
En consecuencia, el objetivo principal del estudio fue analizar las habilidades cognitivas que promueven los textos escolares, edición 2019, de Historia, Geografía y Ciencias Sociales de sétimo y octavo año de enseñanza básica chilena y su correspondencia con las habilidades declaradas en las Bases Curriculares vigentes (2015) y literatura especializada. Tal propósito intenta responder por medio de las siguientes preguntas de investigación: ¿qué tipo de habilidades cognitivas se promueven en los textos escolares de sétimo y octavo básico en la asignatura de Historia, Geografía y Ciencias Sociales? ¿Existe alguna relación entre los textos escolares y las Bases Curriculares de la enseñanza básica para promover el desarrollo de habilidades cognitivas en los estudiantes?

\section{MATERIALES Y MÉTODOS}

El método utilizado de acuerdo con Johnson y Onwuegbuzie (2004) corresponde al tipo de investigación integrativa. Incorpora elementos del método cualitativo y cuantitativo, como lo son aspectos de diseños específicos, como el hermenéutico y descriptivo; análisis de documentos, estadística descriptiva, pautas, patrones de conductas, procesamientos de datos, citas o fragmentos y categorías.

El objeto de estudio fue el texto escolar (tabla 1) que es un recurso pedagógico que registra o expone saberes que debieran ser aprendidos por el estudiantado. Se observa que las actividades educativas intentan promover habilidades, conocimientos, destrezas, actitudes, teorías curriculares, ideologías, discursos que ejercen control en el proceso de aprendizaje e influyen en el comportamiento de los estudiantes y contribuyen con la calidad de la educación (Mineduc, 2015). Se analizó el texto escolar de sétimo y octavo año que repartió en el 2019, de manera gratuita, el Ministerio de Educación chileno a once mil escuelas de educación básica, esto equivale al $90 \%$ del estudiantado chileno.

TABLA 1

Textos estudiados

\begin{tabular}{cccc} 
Autores (año) & Título & Editorial \\
\hline Landa, L. y Pinto, V. (2019a) & Historia, Geografía y Ciencias Sociales $7^{\circ}$ año, texto del estudiante. & SM \\
Landa, L. y Pinto, V. (2019b) & Historia, Geografía y Ciencias Sociales $8^{\circ}$ año, texto del estudiante. & SM \\
\hline
\end{tabular}

Fuente: elaboración propia.

Para identificar las habilidades cognitivas, consignadas en las actividades educativas que orientan el aprendizaje de los estudiantes en los textos escolares se realizó: primero, un análisis de contenidos de las Bases Curriculares para la Educación Básica y los objetivos para la asignatura de Historia, Geografía y Ciencias Sociales. También, se efectuó una revisión de documentos científicos sobre tipos de habilidades cognitivas y, a partir de esos análisis, se construyó un listado de habilidades cognitivas generales y específicas. El instrumento definitivo para pesquisar los datos de los textos escolares, quedó estructurado por cuatro tipos de habilidades cognitivas generales: descriptivas, analíticas, críticas y creativas.

La validez de contenido de la lista de habilidades se llevó a cabo por medio de la técnica juicio de expertos. Se consultó a tres especialistas del área de Ciencias Sociales, quienes dieron cuenta, en una matriz de doble entrada, de la concordancia entre las habilidades específicas y la habilidad general. La opinión de los expertos demostró la total atingencia entre las habilidades generales y específicas. Solo se constató discrepancia en la clasificación de la habilidad "descriptiva".

Una vez validado el listado de habilidades, se construyó una tabla de contingencia que se estructuró en una (1) columna y tres (3) filas. En la columna se registran las habilidades específicas (25) y, en la fila de tres casilleros, las habilidades cognitivas generales (3). Este procedimiento permitió clasificar las habilidades 
que se declaran en los textos. Con esta base, se identificó, valoró y asignó una clasificación a cada una de las habilidades, las cuales se discutieron a partir de las sugerencias realizadas por los expertos.

Los datos obtenidos fueron ingresados al programa SPSS, versión 28 para realizar un análisis descriptivo por frecuencias. Posteriormente, se realizó un análisis de contenido por medio del software Atlas ti 7.5, el cual permitió el procesamiento y el análisis de las habilidades en términos cualitativos. Este software agiliza las actividades implicadas en el proceso de análisis de contenido, como la segmentación del texto en citas, el proceso mismo de codificación, la escritura de memos y anotaciones, así como la realización de vínculos entre citas, memos y códigos (Flores, 2009).

\section{DISCUSIÓN DE LOS RESULTADOS}

Los resultados del análisis de las habilidades de pensamiento que subyacen en las actividades de aprendizaje declaradas en los textos se sintetizan en tablas y figuras, las cuales dan cuenta de nombres, frecuencias, medias, porcentajes, entre otras, con que se promueven. Se ordenan de acuerdo con la siguiente secuencia: a) habilidades cognitivas presentes en las Bases Curriculares para la Educación Básica (2015); b) frecuencia de habilidades presentes en los textos escolares de Historia, Geografía y Ciencias Sociales de sétimo y octavo año; c) habilidades que, según la literatura, deben estar presentes en la enseñanza de la Historia y Geografía en la Educación Básica y con qué frecuencia se hallan en los textos escolares; d) habilidades identificadas con mayor frecuencia en los textos; e) habilidades declaradas por el Mineduc (2015) necesarias de promover en la enseñanza de la disciplina y su presencia en los textos. Los resultados son representados en tablas y figuras.

\section{Habilidades presentes en las Bases Curriculares de Historia, Geografía y Ciencias Sociales}

El cuadro 1 expone las habilidades cognitivas declaradas en las Bases Curriculares de Historia, Geografía y Ciencias Sociales. Las Bases estipulan cuáles son los objetivos de aprendizaje que deben lograr todos los establecimientos escolares de la nación en cada nivel y asignatura (Mineduc, 2020). Producto del análisis de contenido de estos documentos, se pudo identificar 25 habilidades, las cuales se agruparon según sus características en descriptivas (4), analíticas (9), críticas (5) y creativas (7).

Se constató que prácticamente todas las habilidades cognitivas declaradas en las Bases Curriculares están presentes en los textos escolares de Historia, Geografía y Ciencias Sociales. Solo la habilidad "usar herramientas geográficas" no se observó tanto en sétimo como en octavo año. También hubo habilidades no observadas en sétimo como: "Identificar y proponer soluciones a problemas", "representar secuencias cronológicas", "discutir datos, ideas de manera oral y escrita" y "definir conceptos", esta última, no observada en octavo año exclusivamente.

\section{Medias de las dimensiones de habilidades cognitivas en función del curso}

En la tabla 2 se observa el valor de las medias y los porcentajes obtenidos en las dimensiones de las habilidades cognitivas en los textos escolares. Se obtuvo que la media más alta corresponde al tipo de habilidades denominadas creativas $(57,5 \%)$, seguida de la dimensión habilidades analíticas $(47,0 \%)$ y, finalmente, las dimensiones habilidades descriptivas $(42,0 \%)$ y críticas $(25,0 \%)$. La secuencia en el valor de las medias para las habilidades cognitivas es igual, tanto para sétimo como para octavo año. El valor 
de las medias en las dimensiones de las habilidades cognitivas creativas y críticas es superior en octavo que lo observado en sétimo año.

TABLA 2

Habilidades cognitivas presentes en las Bases Curriculares de Historia, Geografía y Ciencias Sociales en Educación Básica, sétimo y octavo año.

\begin{tabular}{|c|c|}
\hline Habilidades & Descripción \\
\hline Descriptivas & $\begin{array}{l}\text { Observar objetos, fenómenos y eventos. } \\
\text { Describir situaciones, hechos, eventos. } \\
\text { Dibujar. } \\
\text { Definir conceptos. }\end{array}$ \\
\hline Analíticas & $\begin{array}{l}\text { Clasificar datos, situaciones, hechos. } \\
\text { Comparar datos, ideas, hechos. } \\
\text { Hacer inferencias. } \\
\text { Formular y contrastar hipótesis. } \\
\text { Formular preguntas sobre hechos o fenómenos. } \\
\text { Discutir datos, ideas de manera oral y escrita. } \\
\text { Interpretar datos y evidencias. } \\
\text { Presentar e interpretar tablas o figuras. } \\
\text { Formular y fundamentar predicciones. }\end{array}$ \\
\hline Críticas & $\begin{array}{l}\text { Plantear conclusiones. } \\
\text { Promover la reflexión. } \\
\text { Evaluar procesos, hechos, resultados, estudios. } \\
\text { Identificar y proponer soluciones a problemas. } \\
\text { Analizar y trabajar con fuentes de consultas. }\end{array}$ \\
\hline Creativas & $\begin{array}{l}\text { Proponer posibles explicaciones. } \\
\text { Planificar y conducir una investigación. } \\
\text { Promover la imaginación. } \\
\text { Promover la creatividad. } \\
\text { Comunicar hallazgos/conocimientos de investigaciones. } \\
\text { Representar secuencias cronológicas. } \\
\text { Aplicar conocimientos en contexto nuevo. }\end{array}$ \\
\hline
\end{tabular}

Los resultados dan cuenta de 343 habilidades presentes en los textos escolares de Historia y Geografía de sétimo y octavo año básico. La mayor cantidad de habilidades se clasificaron como de tipo creativas (115). Por ejemplo, "Crea un esquema que explique la importancia de las ciudades para el surgimiento de las primeras civilizaciones" (T.7 , p. 55), "Imagina cómo sería la vida cotidiana de un indígena bajo la encomienda o de un inquilino en la hacienda. Luego, elabora un testimonio en primera persona agregando los elementos del contexto en el cual se desarrolla" (T.7 ${ }^{\circ}$, p. 121). Luego las analíticas (94) como por ejemplo: "¿Por qué piensan que la agricultura fue tan importante para el origen de las primeras civilizaciones?, ¿qué importancia tiene en el presente esta actividad? (T.8 ${ }^{\circ}$, p. 53); "Infieran nuevas ideas a partir de relaciones entre ideas y elementos de cada fuente y entre ellas.

Por ejemplo, en la fuente $\mathrm{F}$ se puede inferir lo siguiente: se trata de un almacén costero de esclavos traídos en barco; estos se hallan en una situación desmedrada; el hombre blanco es un comprador que elige esclavos. (T.8 , p. 101); descriptivas (84) ejemplos: “Describe las imágenes de estas páginas y señala: ¿qué tipo de fuentes son?, ¿qué transmiten respecto de las ideas o visiones medievales? (T. $7^{\circ}, \mathrm{p}$. 133), “Observen las pinturas, dibujos y grabados de estas páginas (fuentes $A, C$ y E). Luego, respondan: ¿las actividades extractivas se realizaban en áreas urbanas o rurales?, ¿a qué creen que se debía esta situación?" (T.8, p. 99) y críticas (50) ejemplos: "A su juicio, ¿qué características de la civilización europea occidental están presentes en la sociedad a la cual pertenecen?, ¿cuáles dirían que no?, ¿por qué? (T.7, p. 125), “Cómo evaluarías el acceso a la salud y la educación en tu región?, ¿en qué aspectos crees que deberían mejorar? Argumenta" (T.8, p. 213). 
En relación con el nivel educativo, las habilidades cognitivas observadas en los textos escolares en sétimo fueron 165, en cambio, en octavo año suman 178. En sétimo las habilidades de tipo creativas y analíticas en los textos suman más que en octavo año. En cambio, en octavo, se encontraron más habilidades de tipo críticas que en sétimo. Finalmente, la cantidad de habilidades cognitivas de tipo descriptivas es similar en ambos niveles educativos (tabla 3).

TABLA 3

Medias y porcentajes de las dimensiones de habilidades cognitivas, según curso

\begin{tabular}{lccc}
\multicolumn{1}{c}{ Dimensiones } & $\begin{array}{c}\text { Sétimo } \\
\text { Media (\%) }\end{array}$ & $\begin{array}{c}\text { Octavo } \\
\text { Media (\%) }\end{array}$ & $\begin{array}{c}\text { Total } \\
\text { Media (\% Cant.) }\end{array}$ \\
\hline Habilidades Descriptivas & $42,0(12,2)$ & $42,0(12,2)$ & $42,0(24,4: 84)$ \\
Habilidades Analíticas & $50,0(14,6)$ & $44,0(12,8)$ & $47,0(27,4: 94)$ \\
Habilidades Críticas & $22,0(6,4)$ & $28,0(8,2)$ & $25,0(14,6: 50)$ \\
Habilidades Creativas & $51,0(14,9)$ & $64,0(18,7)$ & $57,5(33,6: 115)$ \\
Total valor absoluto & 165 & 178 & 343 \\
\hline
\end{tabular}

Fuente: elaboración propia.

\section{Frecuencia de habilidades cognitivas descriptivas presentes en los textos}

En la Figura 1 se puede constatar las habilidades descriptivas presentes en los textos escolares de Historia y Geografía. Observar objetos, fenómenos y eventos es la habilidad básica más frecuente (24) en octavo y 17 en sétimo año. Como también, describir situaciones, hechos (23) en sétimo y 16 en octavo. En cambio, las habilidades con menor presencia en los textos es definir conceptos (0) en octavo y uno en sétimo.

Figura 1. Frecuencia de habilidades descriptivas en textos escolares de Historia, Geografía y CS.

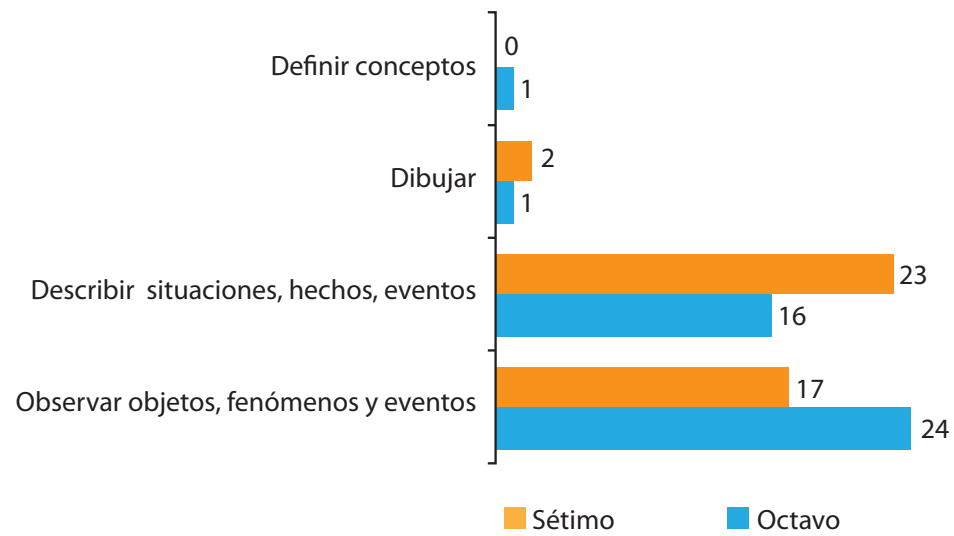

Fuente: elaboración propia.

La Figura 2 presenta los resultados de las habilidades cognitivas según la dimensión de tipo analítica en textos escolares. Lo primero, todas las habilidades expuestas en las bases curriculares están presentes en los textos escolares. Resulta notable que la mayor frecuencia de las habilidades constatadas en esta dimensión llega a catorce, que corresponde a clasificar datos, situaciones y hechos, en octavo año básico; en cambio, la habilidad en sétimo llega a la mitad de esa cantidad. Comparar datos, ideas y hechos tiene una cifra similar en sétimo y octavo (12 y 11), respectivamente. Discutir ideas es una habilidad promovida muy poco en el texto de octavo año (1) no así, en sétimo (8). 
Figura 2. Frecuencia de habilidades analíticas en textos escolares de Historia, Geografía, Ciencias Sociales.

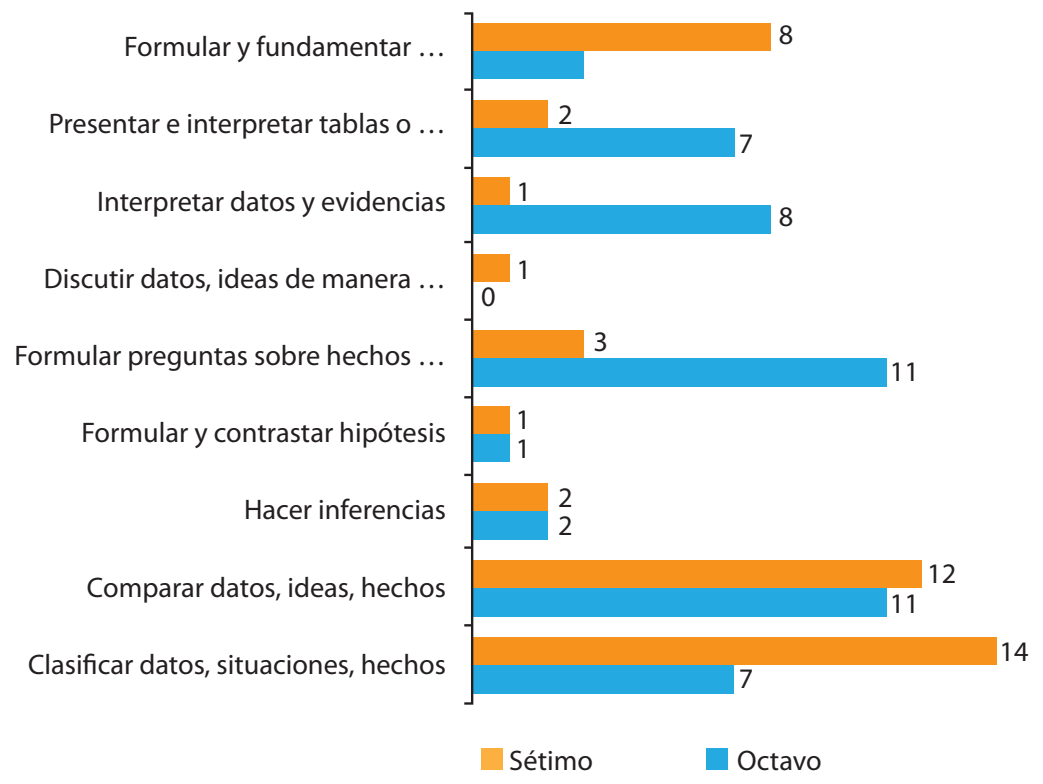

Fuente: elaboración propia.

Si bien se advierte que todas las habilidades críticas correspondientes a la dimensión crítica están presentes en los textos, con excepción de "usar herramientas geográficas" (Figura 3), se observa que cinco de seis habilidades no superan la frecuencia de seis. Asimismo, se percibir también, que la habilidad crítica más frecuente es analizar y trabajar con fuentes de consultas (15) en octavo, y ocho (8) en sétimo.

Figura 3. Frecuencia de habilidades críticas en textos escolares de Historia, Geografía, Ciencias Sociales.

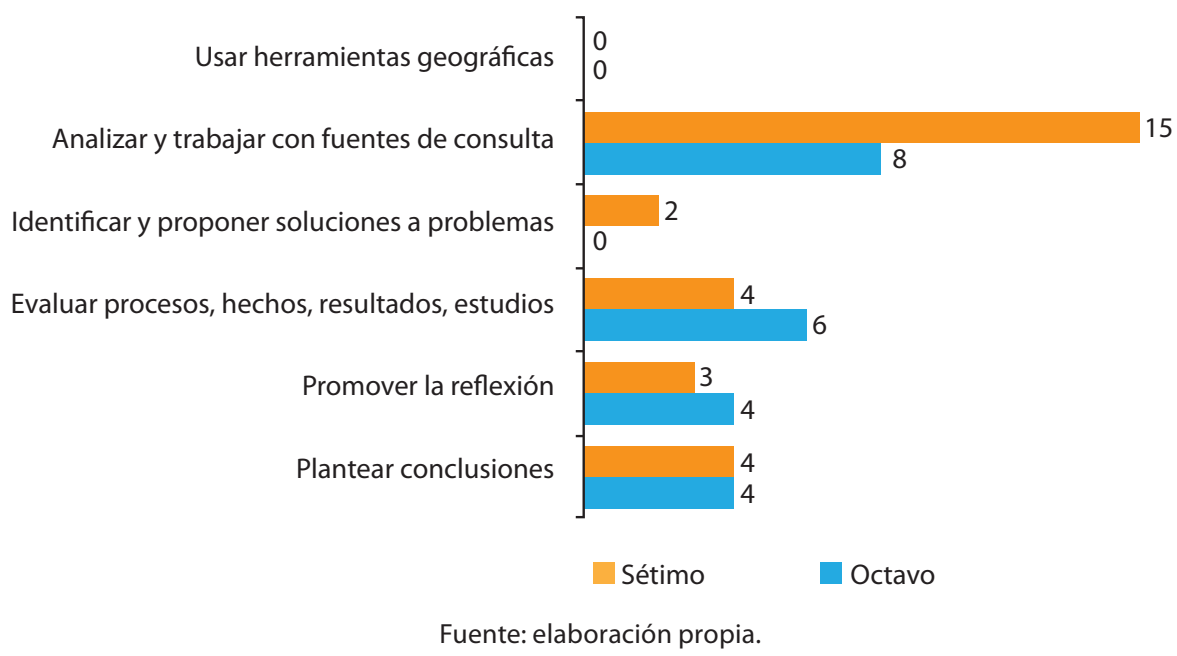

La Figura 4 permite evidenciar la frecuencia de las habilidades específicas de tipo creativas observadas en los textos escolares por nivel educativo. Destaca con mayor constancia las habilidades "aplicar conocimientos en contexto nuevo" (20) en sétimo año, y 17 en octavo año. La habilidad "proponer posibles explicaciones" en sétimo año tiene una frecuencia de 23 y 15 en octavo año. En las cinco restantes habilidades específicas se advierten frecuencias que fluctúan entre uno y once. Finalmente, la habilidad "representar secuencias cronológicas" es de menor frecuencia (2). 
Figura 4. Frecuencia de habilidades creativas en textos escolares de Historia, Geografía, Ciencias Sociales.

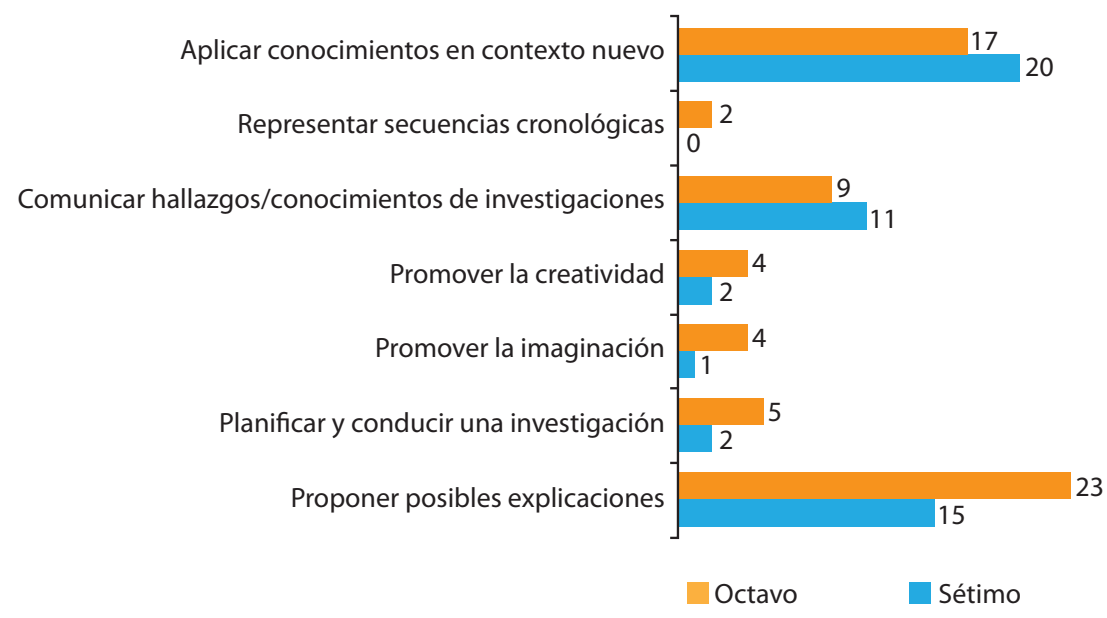

Fuente: elaboración propia.

De la Figura 5 se desprende que las habilidades cognitivas, declaradas por el Ministerio de Educación chileno (2012) para sétimo y octavo año básico, tienen el siguiente nivel de presencia: las habilidades muy presentes en los textos son observar, explicar, proponer explicaciones y aplicar conocimientos. En cambio, las habilidades menos presentes observadas son dibujar, definir conceptos, discutir datos, promover la imaginación, formular y contrastar hipótesis, representar secuencias cronológicas, identificar y proponer soluciones a problemas. Por otro lado, las habilidades denominadas como presentes son las siguientes: comunicar hallazgos/conocimientos, analizar y trabajar con fuentes, clasificar datos, situaciones y hechos, comparar, planificar y conducir una investigación, evaluar y formular preguntas. No fue posible constatar la presencia de la habilidad "usar herramientas geográficas en los textos".

Figura 5. Nivel de presencia de habilidades cognitivas en los textos de Historia, Geografía y Ciencias Sociales

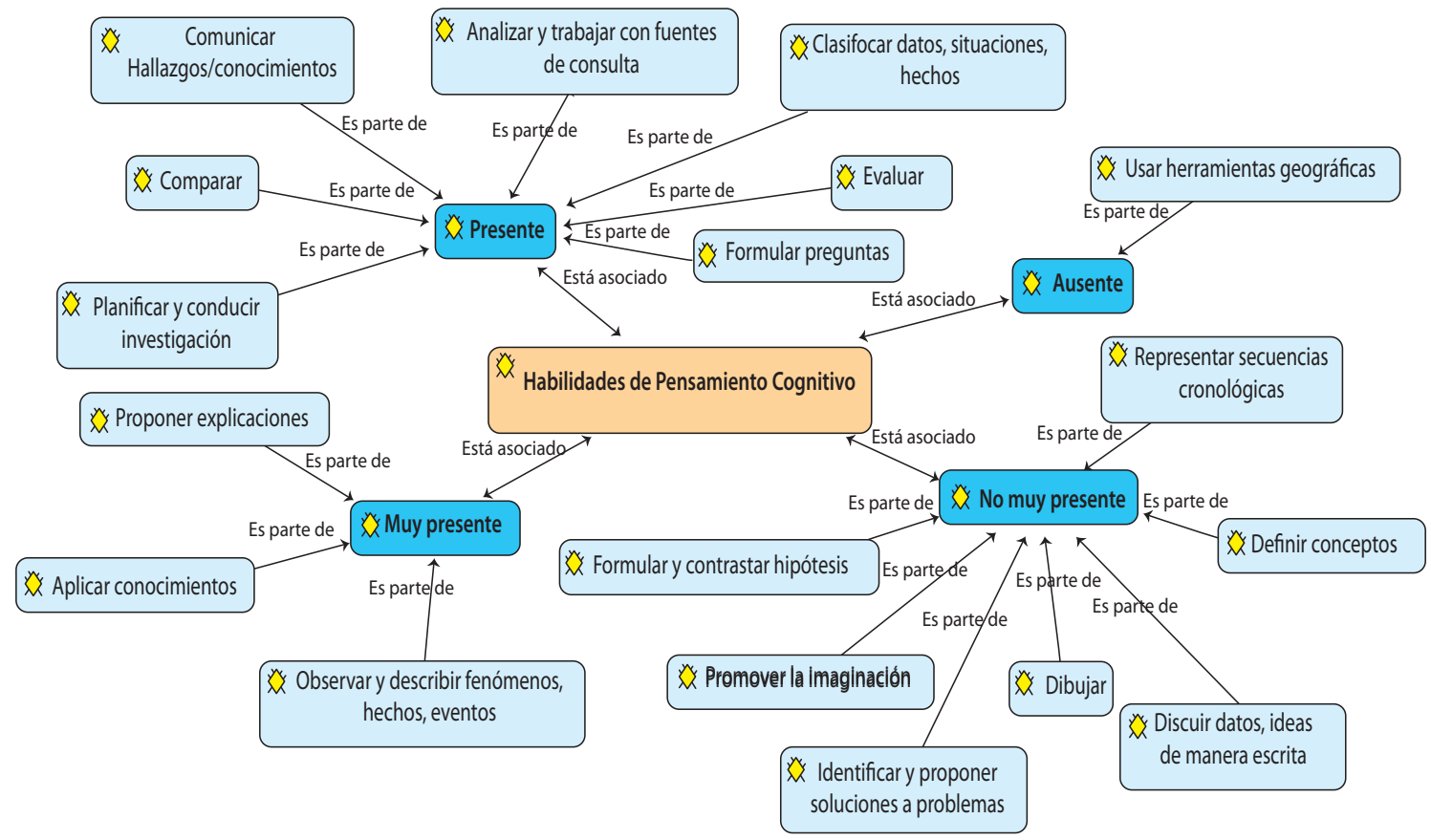

Fuente: elaboración propia a partir de lo declarado por el MINEDUC 2012. 
Según la Tabla 4, la habilidad de pensamiento cognitivo "Observar y describir objetos, fenómenos y eventos" es la que presenta mayor frecuencia en los textos escolares de Historia, Geografía y Ciencias Sociales, seguida de la habilidad "describir situaciones, hechos, eventos", "proponer posibles soluciones" y "aplicar conocimientos en contextos nuevos".

TABLA 4

Habilidades cognitivas que marcan mayor frecuencia en los textos escolares de Historia, Geografía y Ciencias Sociales de sétimo y octavo año básico.

\begin{tabular}{lc}
\multicolumn{1}{c}{ Habilidades cognitivas } & $\begin{array}{c}\text { Mayores frecuencias de habilidades } \\
\text { cognitivas en los textos de Historia... }\end{array}$ \\
\hline Observar y describir objetos, fenómenos y eventos. & 41 \\
Describir situaciones, hechos, eventos. & 39 \\
Proponer posibles explicaciones. & 38 \\
Aplicar conocimientos en contexto nuevo. & 37 \\
Analizar y trabajar con fuentes de consultas & 23 \\
Comparar datos, ideas y hechos. & 23 \\
Clasificar datos, situaciones. & 21 \\
Comunicar hallazgos. & 20 \\
\hline
\end{tabular}

Fuente: elaboración propia.

A través de la lectura de los datos encontrados, se organizaron algunos centros conceptuales que pueden ayudar al logro del objetivo de la presente investigación que fue el analizar las habilidades cognitivas que promueven los textos escolares del estudiante de Historia, Geografía y Ciencias Sociales de sétimo y octavo año de enseñanza básica chilena (edición 2019) y su correspondencia con las habilidades declaradas en las Bases Curriculares (2015).

En la lectura de las figuras y tablas que agrupan los resultados se identificaron cuatros ámbitos de desarrollo pedagógico relativas a las habilidades: descriptivas, analíticas, críticas y creativas, cuyo énfasis formativo cambia entre sí, tanto en cada curso como en las asignaturas en estudio. Situación esperada en un proceso de enseñanza y aprendizaje de ciencias, con el fin de lograr sus objetivos de formación curricular en los planes de estudio del citado nivel de enseñanza. El propósito está declarado en las bases curriculares de estas asignaturas, cuya situación se refleja en estos textos.

En ese sentido, identificar y verificar las habilidades cognitivas que promueven estos textos escolares ayuda a planificar y evaluar el impacto que producen estos libros en el aprendizaje; por lo tanto, se convierte en un factor de apoyo en una eventual retroalimentación formativa al trabajo del profesorado frente a sus estudiantes. Todo esto fortalece la aplicación de instrumentos asociados a una evaluación formativa del aprendizaje escolar.

Ahora bien, no se debe olvidar que el uso de textos escolares tiene una finalidad de apoyo al proceso de aprendizaje y enseñanza, como es el desarrollo de habilidades cognitivas de los alumnos, situación que se reconoce en la presente investigación. Sin embargo, su intensidad es diferenciada en estas habilidades cognitivas descritas, lo cual puede ayudar al profesor/a de aula a identificar su potencial aporte en el logro de aprendizajes específicos de su alumnado, dando oportunidad a una mayor ejercitación, comprensión o refuerzo de las áreas complejas de dicha enseñanza; pues se ha cuantificado el énfasis formativo que tienen estos textos.

Sin embargo, tales resultados pueden ser problematizados desde diferentes perspectivas, ya sea desde una mirada crítica o reproductiva del quehacer pedagógico. Por otro lado, asumir la mirada reproductiva de la enseñanza equivale a afirmar que los citados textos cumplen con los estándares para lo cual fueron 
diseñados; pues promueven los objetivos pedagógicos deseados en su elaboración. Esta situación que se ha confirmado con el presente estudio; sin embargo, los cuyos resultados muestran diferentes énfasis formativos que un profesor de aula debería conocer antes de proceder a su aplicación en la sala de clases.

Tal vez, proponer estos resultados en un programa de formación de profesores en este ámbito disciplinario puede ser una interesante materia de discusión; pues se pueden confrontar dos perspectivas, una ligada a la reproducción y otra a la creatividad (Sáiz, 2011). Cabe señalar que la didáctica de la enseñanza entrega sugerencias metodológicas al profesorado sobre la utilización de recursos, pero no siempre alude a la fortaleza y las limitaciones que dichos instrumentos tienen en la sala de clase, como son los textos escolares. Tal contexto determina la importancia del presente estudio. El texto escolar es claramente un dispositivo instrumental de orden didáctico, el cual se ideó en función de un programa de estudio y, frecuentemente, de una disciplina escolar (Sáiz, 2013). Una disciplina (o materia) escolar no puede confundirse con una disciplina científica. No obstante, en virtud de las orientaciones del currículum, este puede adoptar un enfoque interdisciplinario, destinado a asociar varias disciplinas al mismo tiempo (Lenoir, Lebrun y Hasni, 2012, p. 14).

Al recordar la naturaleza del texto escolar queda en evidencia la función de mediación que ejerce entre el programa y los docentes; así como la función de apoyo a la enseñanza, ya sea en la preparación de las actividades de enseñanza y aprendizaje; pues pretende ser un apoyo efectivo al aprendizaje de los alumnos, entre otras funciones (Lenoir, Lebrun y Hasni, 2012).

Sin embargo, hay un aspecto ideológico del texto escolar que suele pasar inadvertido en la labor docente, como es la presencia de valores y la visión hegemónica de la política pública vigente, lo cual se refleja en el contenido. Para Soaje de Elías (2018), en los textos escolares de Historia se esconde una mirada que denota una falta de profundidad en el tratamiento de los contenidos, una carencia de equidad respecto a los temas tratados y un sesgo ideológico existente en los manuales, lo cual redunda en perjuicio de la objetividad a la que aspiran las Bases Curriculares.

En su análisis, esta autora se plantea algunas interrogantes: ¿cuál es la concepción de Historia y de Ciencia que queda implícita en esta premisa? ¿Cuál es el sentido de la uniformidad,por la tanto, todas las personas estudiantes deben compartir el mismo texto de estudio? ¿Por qué el estudinatado debería estudiar esta asignatura a partir de estos textos y no de otros diferentes? Si uno de los objetivos principales es estimular el pensamiento crítico, ¿por qué no fomentar el uso de diferentes textos a partir de los cuales habría más posibilidad de encontrar distintas visiones acerca del pasado y la actualidad? ¿Cuáles criterios prevalecen para escoger una visión de la Historia en detrimento de otras? (Soaje de Elías, 2018, p. 79).

Por cierto, esta mirada excede a la mirada epistemológica del presente estudio; pues su análisis se centra en una consideración axiológica-ideológica, con el fin de examinar el formato didáctico y curricular que posee el texto de historia escolar en la formación del estudiantado, pero, al mismo tiempo, se advierte que no hay consideraciones sobre el tipo de habilidades cognitivas que desarrollan estos libros (Sáiz, 2013).

Finalmente, estos resultados deben ser entendidos en una visión analítica e interna del texto escolar, cuando se propicia su utilización en la sala de clase, al facilitar una nueva mirada sobre el potencial impacto que ofrece en el desarrollo de habilidades cognitivas de los estudiantes (Lenoir, Lebrun y Hasni, 2012).

\section{SÍNTESIS Y REFLEXIONES FINALES}

Para concluir y de acuerdo con los resultados obtenidos, se presentan a continuación las tendencias más importantes que surgieron en el transcurso del estudio sobre habilidades cognitivas que se promueven en los textos escolares de Historia, Geografía y Ciencias Sociales, en la educación básica. 
Los resultados obtenidos permiten concluir que, en las actividades educativas declaradas en los textos escolares, subyacen habilidades cognitivas del tipo: descriptivas, analíticas, críticas y creativas. Del mismo modo, se evidencia la correspondencia entre los tipos de habilidades cognitivas declaradas en las Bases Curriculares para la Educación básica chilena, y las habilidades cognitivas presentes en las actividades educativas, registradas en los textos escolares.

La capacidad de promover explicaciones, planificar, conducir una investigación, imaginar, crear nuevas ideas, recomponer o armar datos, comunicar hallazgos de investigaciones, representar secuencias cronológicas, aplicar conocimientos en contextos nuevos, son las habilidades características de la dimensión cognitiva denominada creatividad, justamente esta última destreza se observó con mayor frecuencia en las actividades educativas registradas en los textos escolares de Historia, Geografía y Ciencias Sociales. La dimensión creativa adquiere cada vez más relevancia en el ámbito escolar y la escuela creativa ha de formar estudiantes en todas sus dimensiones. Desde el punto de vista educativo, se puede afirmar que la creatividad constituye una base sobre la cual se puede apoyar la enseñanza y el aprendizaje de cualquier disciplina.

Junto con lo anterior, también es posible concluir que de acuerdo con el nivel educativo, los tipos de habilidades cognitivas promovidas en los textos, no registran diferencias importantes en términos de frecuencia de promoción. Sin embargo, en sétimo año se pudo constatar el registro de más habilidades cognitivas de tipo creativas, en cambio, en octavo se observan más habilidades de tipo críticas, ambas corresponden al nivel cognitivo superior. Respecto de la habilidad creativa, esta es parte fundamental en la formación de los estudiantes, así como también, es necesaria para generar soluciones innovadoras que respondan a los cambios ambientales, políticos y sociales que los tiempos modernos demandan (Scott, 2015).

Además, fue posible advertir que las habilidades cognitivas que tienen mayor frecuencia en los textos escolares analizados, corresponden a: observar, explicar y aplicar conocimientos. En cambio, las habilidades menos registradas son definir conceptos, discutir datos, promover la imaginación, formular y contrastar hipótesis, representar secuencias cronológicas, identificar y proponer soluciones a problemas.

Sáez (2017), respecto de la enseñanza de la Historia en los libros de texto de Educación Básica en Chile, encuentra que, en estudiantes de quinto y sexto año de la enseñanza básica, no hay referencia al desarrollo de las habilidades cognitivas que supone la aplicación del texto escolar. Por lo tanto, el presente estudio puede insertarse en esta línea de investigación didáctica y ofrecer una mirada complementaria a dicha problemática.

En este sentido, plantear como relevante una mirada analítica sobre las habilidades cognitivas que subyacen en estos textos escolares puede sugerir una revisión crítica de los ejercicios didácticos en la enseñanza escolar (Sáez-Rosenkranz, 2017). Situación que permitiría, además, una mejor discusión sobre la calidad de la educación que se ofrece en el sistema escolar nacional.

En síntesis, es importante continuar explorando esta línea de investigación y no solo desde las habilidades cognitivas que desarrolla el estudiantado, sino además, en las actividades que facilitan el aprendizaje de la historia por medio del pensamiento histórico (Sáez-Rosenkranz, 2017). Por ello, el uso del texto escolar cumple un rol fundamental en el ámbito escolar como recurso pedagógico y su utilización en la enseñanza puede traer efectos negativos en el aprendizaje, si no se considera potenciar las habilidades cognitivas de orden superior. En este sentido, también es relevante potenciar las funciones que intervienen en el sistema metacognitivo.

Finalmente, cabe recalcar la relevancia de promover las habilidades básicas y las superiores; pues las primeras resultan indispensables en la formación de un pensamiento complejo y se constituyen en un aporte para la comprensión de los procesos formativos desde la perspectiva cognitiva. En este sentido, formar a la ciudadanía del futuro en torno a los ideales de la democracia, el pluralismo y la tolerancia en 
la educación escolar podría significar un desafío mayor, tal como se desarrollará en el Proyecto denominado: "Validación de prototipo escalable de debates en formato online y asincrónico para la formación ciudadana", el cual parte del equipo que planea llevar a cabo en el primer semestre de 2022, con el objetivo de que la comunidad escolar aborde temas del currículum de Historia, Geografía y Ciencias Sociales (específicamente en formación ciudadana y problemas de su interés); con ello se aplican mecanismos orientados a identificar y valorar opiniones o formas de ser diversas y eventualmente divergentes, a pesar del desacuerdo; lo que privilegia contenidos de segundo orden, tal como se manifestó en la Introducción, , de esta manera, que ofrezcan insumos para el desarrollo de nuevas investigaciones en el campo de las ciencias de la educación. Además, las habilidades cognitivas como describir y analizar, son necesarias para la formación del pensamiento crítico en cualquier campo del conocimiento y su ejercicio es escalonado; es decir, debe fomentarse desde la educación básica hasta la educación superior.

\section{REFERENCIAS}

Carretero, M. (2002). Construir y enseñar. Las ciencias sociales y la historia. Buenos Aires: Aique.

Chona, G., Arteta, J., Martínez, S., Ibáñez, X., Pedraza, M. y Fonseca, G. (2006). ¿Qué competencias científicas desarrollamos en el aula? Tecné, Episteme y Didaxis: TED, 20:62-79.

FLORES, R. (2009). Observando observadores: una introducción a las técnicas cualitativas de la Investigación Social. Santiago: Ediciones UC.

Godoy, M. (2015). Las preguntas de docentes como estrategia para el desarrollo de habilidades cognitivas de los estudiantes en la asignatura Historia, Geografía y Ciencias Sociales. Foro Educacional, 24:57-76.

Gómez, C., Ortuño, J. y Molina, S. (2014). Aprender a pensar históricamente. Retos para la historia en el siglo XXI. Revista Tempo e Argumento, 6(11):5-27.

Johnson, R. B. y Onwuegbuzie, A. J. (2004). Mixed methods research: a research paradigm whose time has come. Educational Researcher, 33(7):14-26.

Landa, L. y Pinto, V. (2019a). Historia, Geografía y Ciencias Sociales, séptimo año. Texto del estudiante. Santiago: SM.

Landa, L. y Pinto, V. (2019b). Historia, Geografía y Ciencias Sociales, octavo año. Texto del estudiante. Santiago: SM.

Lenoir, Y., Lebrun, J., \& Hasni, A. (2016). Análisis de Textos Escolares: Algunos Fundamentos y Desafíos a tener en Cuenta. Revista Iberoamericana de Evaluación Educativa, 5(3):11-30.

Ministerio de Educación (2015). Bases curriculares de Educación $7^{\circ}$ básico a 2 medio. Santiago: Unidad de Currículum y Evaluación. Disponible en: https://media.mineduc.cl/wp-content/uploads/ sites/28/2017/07/Bases-Curriculares-7\%C2\%BA-b\%C3\%A1sico-a-2\%C2\%BA-medio.pdf

Ministerio de EdUCACIÓn (2018). Circular sobre textos escolares y útiles escolares. Superintendencia de Educación. Disponible en: https://www.supereduc.cl/wp-content/uploads/2018/03/circularnormativa-sobre-textos-y-utiles-escolares.pdf

Ministerio de Educación (2020). Textos Escolares SIGE. https://www.ayudamineduc.cl/ficha/textos-escolares-sige-4

Morales, J. (2017). Pensamiento crítico y lectura en ciencias sociales. Revista electrónica Calidad en la Educación Superior, 8(2):265-282.

Moreno, A., Toro., S., y Gómez, F. (2020). Formación inicial de maestros de educación física: conectando un quehacer pedagógico de colonial con la intervención social, política e insurgente del espacio público. RETOS: Nuevas Tendencias en Educación Física, Deporte y Recreación, 37:605-612. 
Peña, S., Toro, S., Cárcamo, J., Hernández-Mosqueira, C. y Cresp, M. (2020). La fragmentación del conocer en educación física. RETOS: Nuevas Tendencias en Educación Física, Deporte y Recreación, 39:231-237.

Portillo, C. (2017). Educación por habilidades: Perspectivas y retos para el sistema educativo. Revista Educación, 41(2):118-130.

Romero, Y., y TAPIA, F. (2014). Desarrollo de las habilidades cognitivas en niños de edad escolar. Multiciencias, 14(1):297-303.

Sáez-Rosenkranz, I. (2017). La enseñanza de la historia en los libros de texto de Educación Básica en Chile. Enseñanza de las Ciencias Sociales: revista de investigación, 16:27-40.

SÁlz, S. (2013). Alfabetización histórica y competencias básicas en libros de texto de historia y en aprendizaje de los estudiantes. Revista Didáctica de las Ciencias Experimentales y Sociales, 27:43-66.

Sáı, J. (2011). Actividades de libros de texto de Historia, competencias básicas y destrezas cognitivas, una difícil relación: análisis de manuales de $1^{\circ}$ y $2^{\circ}$ de ESO. Didáctica de las Ciencias Experimentales y Sociales, 25:37-64.

Scott, C. (2015). El futuro del aprendizaje 2 ¿Qué tipo de aprendizaje se necesita en el siglo XXI? Paris: UNESCO.

SOAJE de Elías, R. (2018). Textos escolares: consideraciones didácticas. Educación y Educadores, 21(1):73-92. 